\title{
Natural Enemies of Cinara cedri Mimeur 1936 (Hemiptera: Aphididae) in Cedar Forests in Isparta Regional Forest Directorate
}

\author{
Şükran OĞUZOĞLU*® , Mustafa AVCIĐ \\ SDU Forest Faculty Isparta, 32260, TURKEY \\ *Corresponding Author: sukranoguzoglu@sdu.edu.tr
}

Received Date: 31.07.2018

Accepted Date: 29.04.2019

\begin{abstract}
Aim of study: This study was performed between 2015 and 2016 with the aim of identifying natural enemies of cedar aphid (Cinara cedri Mimeur, 1936) (Hemiptera: Aphididae) in cedar (Cedrus libani A. Rich.) forests.

Area of study: Specimens were collected from 40 areas in Isparta Regional Forest Directorate cedar forests.

Material and methods: At each site, specimens were randomly collected from infested shoots. Shoots were cut and put in plastic bags and containers and were brought to the laboratory, examined with a stereomicroscope. The adults obtained were prepared and sent to specialists for identification.

Main results: Twenty eight species of insect enemies were found, most of them coccinellid and syrphid predators. Harmonia axyridis Pallas, 1773, Anatis ocellata (Linnaeus, 1758) (Coleoptera: Coccinellidae), Asaphes vulgaris Walker, 1834 (Hymenoptera: Pteromalidae) and Pauesia anatolica Michelena, Assael \& Mendel, 2005 (Hymenoptera: Braconidae) were found for the first time in the study area. Moreover, 17 species were firstly determined as a natural enemy of Cinara cedri.

Highlights: In this study, new records were identified for study area. Moreover, 17 species were firstly determined as a natural enemy of Cinara cedri.

Keywords: Parasitoid, Predator, Cinara cedri, Isparta, Turkey.

\section{Cinara cedri Mimeur 1936 (Hemiptera: Aphididae)'nin Isparta Orman Bölge Müdürlüğü Sedir Ormanlarındaki Doğal Düşmanları}

$\ddot{O} \mathbf{z}$

Çalışmanin amacı: Bu çalışma, Güneybatı Anadolu'da 2015-2016 yıllarında 40 sedir (Cedrus libani A. Rich.) ormanında sedir yaprak bitinin (Cinara cedri Mimeur, 1936) (Hemiptera: Aphididae) doğal düşmanlarını tespit etmek amacıyla gerçekleştirilmiştir.

Çalışma alanı: Örnekler, Isparta Orman Bölge Müdürlüğü sedir ormanlarındaki 40 alandan toplanmıştır.

Materyal ve Yöntem: Örnekler her bir alandaki bulaşık sürgünlerden rastgele toplanmıştır. Sürgünler kesilmiş ve plastik kaplara konulmuş ve laboratuvara getirilerek stereomikroskop ile incelenmiştir. Erginlerin preparasyonu yapılmış ve teşhisleri için uzmanlara gönderilmiştir.

Sonuçlar: Çoğu coccinellid ve syrphid olan 28 adet doğal düşman bulunmuştur. Harmonia axyridis Pallas, 1773, Anatis ocellata (Linnaeus, 1758) (Coleoptera: Coccinellidae), Asaphes vulgaris Walker, 1834 (Hymenoptera: Pteromalidae) ve Pauesia anatolica Michelena, Assael \& Mendel, 2005 (Hymenoptera: Braconidae) çalışma sahasında ilk kez tespit edilmiştir. Ayrıca 17 tür Cinara cedri'nin doğal düşmanı olarak ilk kez bulunmuştur.

Önemli vurgular: Çalışma sahası için yeni kayıtlar tespit edilmiştir. Ayrıca 17 türün C. cedri'nin doğal düşmanı olduğu ilk kez belirlenmiştir.

Anahtar kelimeler: Parazitoit, Predatör, Cinara cedri, Isparta, Türkiye. 


\section{Introduction}

Taurus cedar (Cedrus libani A. Rich.) is one of the most important forest tree species in Turkey. Taurus cedar locates naturally in the Taurus Mountains of Southern Turkey, in Western Syria and in the Lebanon Mountains. The timber of this tree has particular values for many for years, due to the exceptional durability, strength aesthetically pleasing appearance. In Turkey, C. libani covers a land area of 482,391 ha and is located especially in the south of Turkey (OGM, 2015).

It was identified two aphid species (Cinara cedri and C. laportei) on Cedrus libani in Turkey (Aslan \& Uygun, 2005; Aytar, 2006). C. cedri differentiates from $C$. laportei in terms of the number of antenna segments (Blackman \& Eastop, 2012). C. cedri is that the most common cedar aphid in Turkey (Çanakçıŏlu, 1975; Ünal \& Özcan, 2005; Aytar, 2006; Ülgentürk et. al., 2013; Akyıldırım, Şenol, Görür \& Demirtaş, 2013).

Cinara cedri Mimeur 1936 (Hemiptera: Aphididae) was first identified and described in Morocco on Atlas cedar (Cedrus atlantica) (Mimeur, 1935). It causes damage by sucking the sap of cedar shoots and foliage and resulting in desiccation decolourization of needles. Honeydew secreted by the aphids attracts several the other insect species to the trees, and the disease vectored by these species may further damage the trees. The growth of sooty-molds on affected foliage and shoots may reduce tree productivity in terms of both timber and seed yields (NúňezPerez and Tizado, 1996).

C. cedri was firstly identified on C. libani at 1964 in Gaziantep, Turkey (Tuatay \& Remaudiere, 1964) and then was found in many regions of Turkey (Çanakçıoğlu, 1975;
Düzgüneş, Toros, Kılınçer \& Kovanc1, 1980; Usta \& Keskin, 1992; Tuatay, 1999; Uygun, Toros, Ulusoy, Satar \& Özdemir, 2000; Toper Kaygin, Görür \& Çota, 2008; Ünal \& Özcan, 2005; Aytar, 2006; Akyıldırım et. al., 2013). So far, it was determined to feed on $C$. libani, C. deodora, C. atlantica, Cedrus sp., Thuja sp. and Pinus sp. (Çanakçıŏlu, 1975; Ünal \& Özcan, 2005; Aytar, 2006; Ülgentürk et. al., 2013; Aky1ldırım et. al., 2013).

In Turkey, there is one study about natural enemies of C. cedri in forest. In the study, the damage to this species was observed in the plantation forests and it was identified parasitoid of C. cedri in the southeast of Anatolia (Aytar, 2006). In other studies, it was mainly identified natural enemies of $C$. cedri in non-forest areas such as park and gardens. Therefore, this research was conducted to identify the natural enemies associated with $C$. cedri on cedar trees in the southwest of Anatolia.

\section{Material and Methods}

Study area

The work was conducted between 2015 and 2016 in Isparta Regional Forest Directorate, which is located in the Lakes District of southwest Turkey. Lakes District is one of the important regions for Turkey. It was performed $820-1738 \mathrm{~m}$ altitudes, in a total of 40 areas, in both natural stands and plantations of Cedrus libani. Study areas comprise of pure C. libani stands (13), $C$. libani-Pinus nigra subsp. pallasiana mixed stands (25), C. libani-Quercus vulcanica mixed stand (1) and C. libani-Abies cilicica subsp. isaurica stand (1). Information on the study site locations and typical stand tree species composition is given in Table 1 .

Table 1. Characteristics of the areas where Cinara cedri was sampled

\begin{tabular}{llllc}
\hline No & Location & Coordinates & Altitude (m) & $\begin{array}{c}\text { Dominant tree } \\
\text { species* }\end{array}$ \\
\hline 1 & Isparta-Center & $37^{\circ} 46^{\prime} 43^{\prime \prime N}-30^{\circ} 32^{\prime} 49^{\prime \prime} \mathrm{E}$ & 1043 & $\mathrm{a}, \mathrm{b}$ \\
\hline 2 & Isparta-SDÜ Campus & $37^{\circ} 49^{\prime} 49^{\prime \prime N}-30^{\circ} 32^{\prime} 05^{\prime \prime} \mathrm{E}$ & 1019 & $\mathrm{a}, \mathrm{b}$ \\
\hline 3 & Burdur-Bucak-Karapınar & $37^{\circ} 21^{\prime} 46^{\prime \prime N}-30^{\circ} 22^{\prime} 00^{\prime \prime} \mathrm{E}$ & 820 & $\mathrm{a}, \mathrm{b}$ \\
\hline 4 & Burdur-Bucak-Seydiköy & $37^{\circ} 30^{\prime} 27^{\prime \prime N}-30^{\circ} 33^{\prime} 07^{\prime \prime} \mathrm{E}$ & 892 & $\mathrm{a}$ \\
\hline 5 & Isparta-Sağ Âş1k Tomb & $37^{\circ} 21^{\prime} 46^{\prime \prime} \mathrm{N}-30^{\circ} 39^{\prime} 26^{\prime \prime} \mathrm{E}$ & 838 & $\mathrm{a}, \mathrm{b}$ \\
\hline 6 & Isparta-Eğirdir-Barla & $37^{\circ} 55^{\prime} 04^{\prime \prime} \mathrm{N}-30^{\circ} 44^{\prime} 49^{\prime \prime} \mathrm{E}$ & 1182 & $\mathrm{a}, \mathrm{b}$ \\
\hline 7 & Isparta-Eğirdir & $37^{\circ} 52^{\prime} 24^{\prime \prime N}-30^{\circ} 49^{\prime} 36^{\prime \prime} \mathrm{E}$ & 976 & $\mathrm{a}, \mathrm{b}$ \\
\hline
\end{tabular}


Table 1. (Continued)

\begin{tabular}{|c|c|c|c|c|}
\hline No & Location & Coordinates & Altitude (m) & $\begin{array}{c}\text { Dominant tree } \\
\text { species* }\end{array}$ \\
\hline 8 & Isparta-Eğirdir-Beşkuyu & $37^{\circ} 43^{\prime} 44^{\prime \prime N}-30^{\circ} 48^{\prime} 25^{\prime \prime} \mathrm{E}$ & 1738 & $\mathrm{a}$ \\
\hline 9 & Isparta-Güneyce & $37^{\circ} 40^{\prime} 45^{\prime \prime N}-30^{\circ} 45^{\prime} 22^{\prime \prime} \mathrm{E}$ & 880 & $a, b$ \\
\hline 10 & Isparta-Çobanisa & $37^{\circ} 47^{\prime} 49^{\prime \prime N}-30^{\circ} 47^{\prime} 04^{\prime \prime} \mathrm{E}$ & 1716 & $\mathrm{a}$ \\
\hline 11 & Isparta-Gökçay Park & $37^{\circ} 44^{\prime} 49^{\prime \prime N}-30^{\circ} 32^{\prime} 45^{\prime \prime} \mathrm{E}$ & 1153 & $a, b$ \\
\hline 12 & Isparta-Hisartepe & $37^{\circ} 43^{\prime} 45^{\prime \prime N}-30^{\circ} 31^{\prime} 56^{\prime \prime E}$ & 1485 & $a, b$ \\
\hline 13 & Isparta-Yenişarbademli & $37^{\circ} 42^{\prime} 33^{\prime \prime N}-31^{\circ} 20^{\prime} 52^{\prime \prime} \mathrm{E}$ & 1415 & $\mathrm{a}$ \\
\hline 14 & Isparta-Aksu & $37^{\circ} 43^{\prime} 35^{\prime \prime N}-31^{\circ} 12^{\prime} 21^{\prime \prime} \mathrm{E}$ & 1213 & $\mathrm{a}, \mathrm{b}$ \\
\hline 15 & Isparta-Aksu-Yakaafşar & $37^{\circ} 44^{\prime} 36^{\prime \prime N}-31^{\circ} 10^{\prime} 19^{\prime \prime} \mathrm{E}$ & 1275 & $\mathrm{a}, \mathrm{b}$ \\
\hline 16 & Isparta-Aksu-Yaka & $37^{\circ} 43^{\prime} 55^{\prime \prime N}-31^{\circ} 14^{\prime} 08^{\prime \prime} \mathrm{E}$ & 1326 & $a, b$ \\
\hline 17 & Isparta-Atabey & $37^{\circ} 56^{\prime} 45^{\prime \prime N}-30^{\circ} 37^{\prime} 14^{\prime \prime} \mathrm{E}$ & 1041 & $a, b$ \\
\hline 18 & Isparta-Gönen & $37^{\circ} 57^{\prime} 55^{\prime \prime N}-30^{\circ} 31^{\prime} 24^{\prime \prime} \mathrm{E}$ & 1086 & $\mathrm{a}, \mathrm{b}$ \\
\hline 19 & Isparta-Keçiborlu-1 & $37^{\circ} 57^{\prime} 29^{\prime \prime} \mathrm{N}-30^{\circ} 18^{\prime} 03^{\prime \prime} \mathrm{E}$ & 1042 & $\mathrm{a}$ \\
\hline 20 & Isparta-Keçiborlu-2 & $37^{\circ} 54^{\prime} 57^{\prime \prime N}-30^{\circ} 17^{\prime} 28^{\prime \prime} \mathrm{E}$ & 1067 & $\mathrm{a}$ \\
\hline 21 & Isparta-Keçiborlu-Senir & $37^{\circ} 49^{\prime} 17^{\prime \prime N}-30^{\circ} 17^{\prime} 24^{\prime \prime} \mathrm{E}$ & 1026 & $\mathrm{a}$ \\
\hline 22 & Isparta-Senirkent-Kapıdağ & $38^{\circ} 06^{\prime} 00^{\prime \prime} \mathrm{N}-30^{\circ} 46^{\prime} 10^{\prime \prime} \mathrm{E}$ & 1507 & $\mathrm{a}$ \\
\hline 23 & Isparta-Senirce & $37^{\circ} 53^{\prime} 03^{\prime \prime N}-30^{\circ} 30^{\prime} 06^{\prime \prime} \mathrm{E}$ & 1025 & $\mathrm{a}$ \\
\hline 24 & Burdur-Altınyayla-Kumluağıl & $36^{\circ} 50^{\prime} 19^{\prime \prime} \mathrm{N}-29^{\circ} 24^{\prime} 19^{\prime \prime} \mathrm{E}$ & 1604 & $\mathrm{a}$ \\
\hline 25 & Burdur-Altınyayla-Tamtır & $36^{\circ} 50^{\prime} 48^{\prime \prime} \mathrm{N}-29^{\circ} 24^{\prime} 23^{\prime \prime} \mathrm{E}$ & 1564 & $\mathrm{a}$ \\
\hline 26 & Burdur-Çavdır & $37^{\circ} 09^{\prime} 51^{\prime \prime N}-29^{\circ} 42^{\prime} 51^{\prime \prime} \mathrm{E}$ & 1150 & $a, b$ \\
\hline 27 & Burdur-Tefenni & $37^{\circ} 20^{\prime} 47^{\prime \prime N}-29^{\circ} 48^{\prime} 27^{\prime \prime} \mathrm{E}$ & 1138 & $a, b$ \\
\hline 28 & Burdur-Karamanl1 & $37^{\circ} 22^{\prime} 45^{\prime \prime N}-29^{\circ} 52^{\prime} 23^{\prime \prime} \mathrm{E}$ & 1121 & $\mathrm{a}, \mathrm{b}$ \\
\hline 29 & Isparta-Kızıldağ National Park & $38^{\circ} 02^{\prime} 22^{\prime \prime N}-31^{\circ} 21^{\prime} 52^{\prime \prime} \mathrm{E}$ & 1421 & $\mathrm{a}$ \\
\hline 30 & Isparta-Çarıksaraylar & $38^{\circ} 07^{\prime} 43^{\prime \prime N}-31^{\circ} 25^{\prime} 28^{\prime \prime} \mathrm{E}$ & 1301 & $\mathrm{a}, \mathrm{b}$ \\
\hline 31 & Isparta-Yalvaç-City Forest & $38^{\circ} 18^{\prime} 58^{\prime \prime} \mathrm{N}-31^{\circ} 10^{\prime} 36^{\prime \prime} \mathrm{E}$ & 1116 & $a, b$ \\
\hline 32 & Isparta-Yalvaç-Bağkonak & $38^{\circ} 12^{\prime} 31^{\prime \prime N}-31^{\circ} 15^{\prime} 57^{\prime \prime E}$ & 1136 & $\mathrm{a}, \mathrm{b}$ \\
\hline 33 & Burdur-Bucak-Katran Mountain & $37^{\circ} 21^{\prime} 54^{\prime \prime N}-30^{\circ} 05^{\prime} 38^{\prime \prime} \mathrm{E}$ & 1136 & $\mathrm{a}, \mathrm{c}$ \\
\hline 34 & Isparta-Büyükgökçeli & $37^{\circ} 53^{\prime} 13^{\prime \prime N}-30^{\circ} 44^{\prime} 04^{\prime \prime} \mathrm{E}$ & 1047 & $\mathrm{a}, \mathrm{b}$ \\
\hline 35 & Isparta-Uluborlu & $38^{\circ} 03^{\prime} 58^{\prime \prime N}-30^{\circ} 25^{\prime} 09^{\prime \prime} \mathrm{E}$ & 1150 & $\mathrm{a}$ \\
\hline 36 & Isparta-Ayazmana Park & $37^{\circ} 44^{\prime} 48^{\prime \prime N}-30^{\circ} 34^{\prime} 54^{\prime \prime} \mathrm{E}$ & 1045 & $\mathrm{a}, \mathrm{b}$ \\
\hline 37 & $\begin{array}{l}\text { Isparta-Eğirdir-Kasnak Oak } \\
\text { Nature Protected Area }\end{array}$ & $37^{\circ} 42^{\prime} 46^{\prime \prime} \mathrm{N}-30^{\circ} 50^{\prime} 11^{\prime \prime} \mathrm{E}$ & 1192 & $\mathrm{a}, \mathrm{d}$ \\
\hline 38 & Isparta-Gölcük Nature Park & $37^{\circ} 43^{\prime} 45^{\prime \prime N}-30^{\circ} 29^{\prime} 05^{\prime \prime} \mathrm{E}$ & 1400 & $\mathrm{a}, \mathrm{b}$ \\
\hline 39 & Isparta-Senirkent & $38^{\circ} 06^{\prime} 12^{\prime \prime} \mathrm{N}-30^{\circ} 33^{\prime} 27^{\prime} \mathrm{E}$ & 1001 & $\mathrm{a}, \mathrm{b}$ \\
\hline 40 & Isparta-Gelincik & $37^{\circ} 45^{\prime} 36^{\prime \prime N}-30^{\circ} 27^{\prime} 59^{\prime \prime} \mathrm{E}$ & 1255 & $\mathrm{a}, \mathrm{b}$ \\
\hline
\end{tabular}

\section{Collection of Insects}

At each site, specimens were randomly collected on infested shoots of trees. It was sampled about 10 trees when population density of Cinara cedri was high, but it was sampled 1-2 trees when its population density was low. It was controlled if there were any other pests on shoots. Photos of infested shoots and damage of Cinara cedri were taken (Figure 1). Infested shoots were cut and put in plastic bags and containers. Adult coccinellids were collected by hand picking and the methodology described by Steiner (1962). Shoots and specimens were transferred to the laboratory and examined with a stereomicroscope. They were made preparations of the aphids and they were identified according to Blackman \&Eastop (2012) (Figure 2). Specimens in their immature stage were put in different sizes of culture containers covered with a net. Containers were periodically controlled to emerge adults of parasitoids and predators. Observations were conducted on the predators' larvae to record feeding on $C$. cedri. The adults obtained were prepared and sent to specialists for identification. Adult Syrphidae were identified according to sex. After the identification, it was researched 
whether they are aphidophagus or not according to the literature.

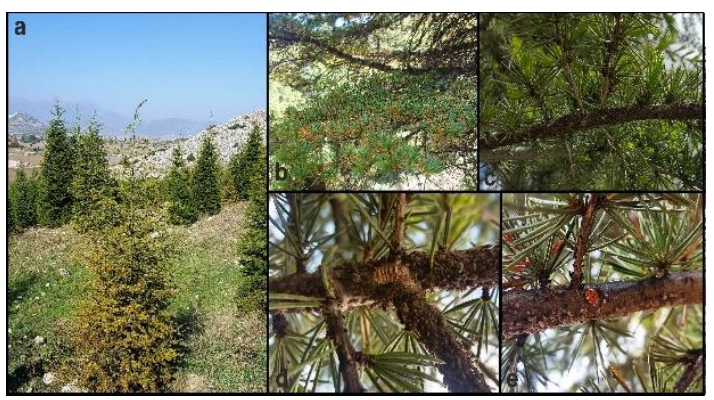

Figure 1. Damage (a, b, c) and natural enemies species $(d, e)$ on infested shoots of Cinara cedri

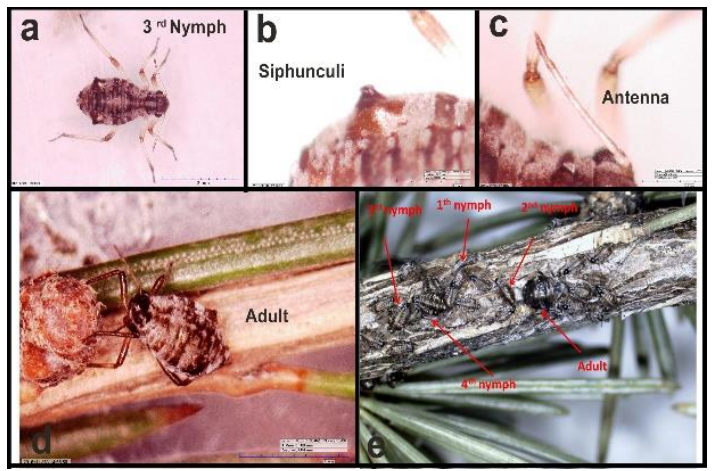

Figure 2. Some morphological characteristics $(\mathrm{b}, \mathrm{c})$ and different stages of Cinara cedri (a, $\mathrm{d}, \mathrm{e})$

Table 2. Natural enemies of Cinara cedri Mimeur 1936 (Hemiptera: Aphididae) in cedar forests of Isparta Forest Regional Directorate

\begin{tabular}{|c|c|c|c|}
\hline Order: Family & Species & Species code & Specimen number \\
\hline \multirow{18}{*}{ Coleoptera: Coccinellidae } & Adalia bipunctata (Linnaeus, 1758) & ADABIP & 21 \\
\hline & Adalia decempunctata (Linnaeus, 1758) & ADADEC & 1 \\
\hline & Adalia fasciatopunctata revelierei Mulsant & ADAFAS & 37 \\
\hline & Anatis ocellata (Linnaeus, 1758) & ANAOCE & 3 \\
\hline & Brumus quadripustulatus Linnaeus, 1758 & BRUQUA & 53 \\
\hline & Chilocorus bipustulatus (Linnaeus, 1758) & CHIBIP & 6 \\
\hline & Coccinella septempunctata Linnaeus, 1758 & COCSEP & 168 \\
\hline & Coccinula quatardecimpustulata (Linnaeus, 1758) & COCQUA & 4 \\
\hline & Exochomus undulatus Weise, 1878 & EXOUND & 1 \\
\hline & Harmonia axyridis Pallas, 1773 & HARAXY & 5 \\
\hline & Harmonia quadripunctata (Pontoppidan, 1763) & HARQUA & 185 \\
\hline & Hippodamia (Hippodamia) variegata Goeze, 1777 & HIPVAR & 5 \\
\hline & Hippodamia undecimnotata (Schneider, 1792) & HIPUND & 2 \\
\hline & Myzia oblongoguttata (Linnaeus, 1758) & MYZOBL & 9 \\
\hline & Oenopia conglobata (Linnaeus, 1758) & OENCON & 5 \\
\hline & Oenopia lyncea (Olivier, 1808) & OENLYN & 2 \\
\hline & Scymnus subvillious (Goeze, 1777) & SCYSUB & 23 \\
\hline & Scymnus pallipediformis Günther, 1958 & SCYPAL & 1 \\
\hline \multirow{5}{*}{ Diptera: Syrphidae } & Sphaerophoria scripta (Linnaeus, 1758) & SPHSCP & 5 \\
\hline & Syrphus ribesii (Linnaeus, 1758) & SYRRIB & 1 \\
\hline & Syrphus vitripennis Meigen, 1822 & SYRVIT & 21 \\
\hline & Eupeodes corollae (Fabricius, 1794) & EUPCOR & 2 \\
\hline & Scaeva pyrastri (Linnaeus, 1758) & SCAPYR & 18 \\
\hline Neuroptera: Chrysopidae & Chrysoperla carnea (Stephens, 1836) & CHRCAR & 4 \\
\hline
\end{tabular}

Results

Natural enemies were obtained from 36 of the 40 stands examined. 846 specimens were collected and a total of 28 insect natural enemy species were identified belonging to six families including 18 coccinellids (Coleoptera), 5 syrphids (Diptera), 2 braconids and 1 pteromalid (Hymenoptera), raphidiid and chrysopid (Neuroptera). These enemies included 3 parasitoids were found (Table 2, Figure 3).

Harmonia axyridis Pallas, 1773 (Coleoptera: Coccinellidae) is a new record for study area. The highest numbers of adults were for Pauesia anatolica Michelena, Assael \& Mendel, 2005 (Hymenoptera: Braconidae), with 248, followed by Harmonia quadripunctata (Pontoppidan, 1763) with 185 and Coccinella septempunctata Linnaeus, 1758 with 168. In contrast, only single adults of Adalia decempunctata (Linnaeus, 1758), Exochomus undulatus Weise, 1878, Scymnus pallipediformis Günther, 1958 (Coleoptera: Coccinellidae) and Syrphus ribesii (Linnaeus, 1758) (Diptera: Syrphidae) were found. 
Kastamonu Uni., Orman Fakültesi Dergisi, 2019, 19 (2):173-185

Kastamonu Univ., Journal of Forestry Faculty

Table 2 (continued)

\begin{tabular}{lllc}
\hline Order: Family & Species & $\begin{array}{l}\text { Species } \\
\text { code }\end{array}$ & $\begin{array}{l}\text { Specimen } \\
\text { number }\end{array}$ \\
\hline Neuroptera: Raphidiidae & $\begin{array}{l}\text { Phaeostigma (Aegeoraphidia) ressli (Aspöck \& Aspöck, } \\
1964)\end{array}$ & PHARES & 2 \\
\hline $\begin{array}{l}\text { Hymenoptera: } \\
\text { Braconidae }\end{array}$ & $\begin{array}{l}\text { Pauesia (Pauesia) anatolica Michelena, Assael \& } \\
\text { Mendel, 2005 }\end{array}$ & PAUANA & 248 \\
\cline { 2 - 4 } & Aphidius sp. & APHSP & 2 \\
\hline $\begin{array}{l}\text { Hymenoptera: } \\
\text { Pteromalidae }\end{array}$ & Asaphes vulgaris Walker, 1834 & ASAVUL & 13 \\
\hline Total specimen number & & & 847 \\
\hline
\end{tabular}

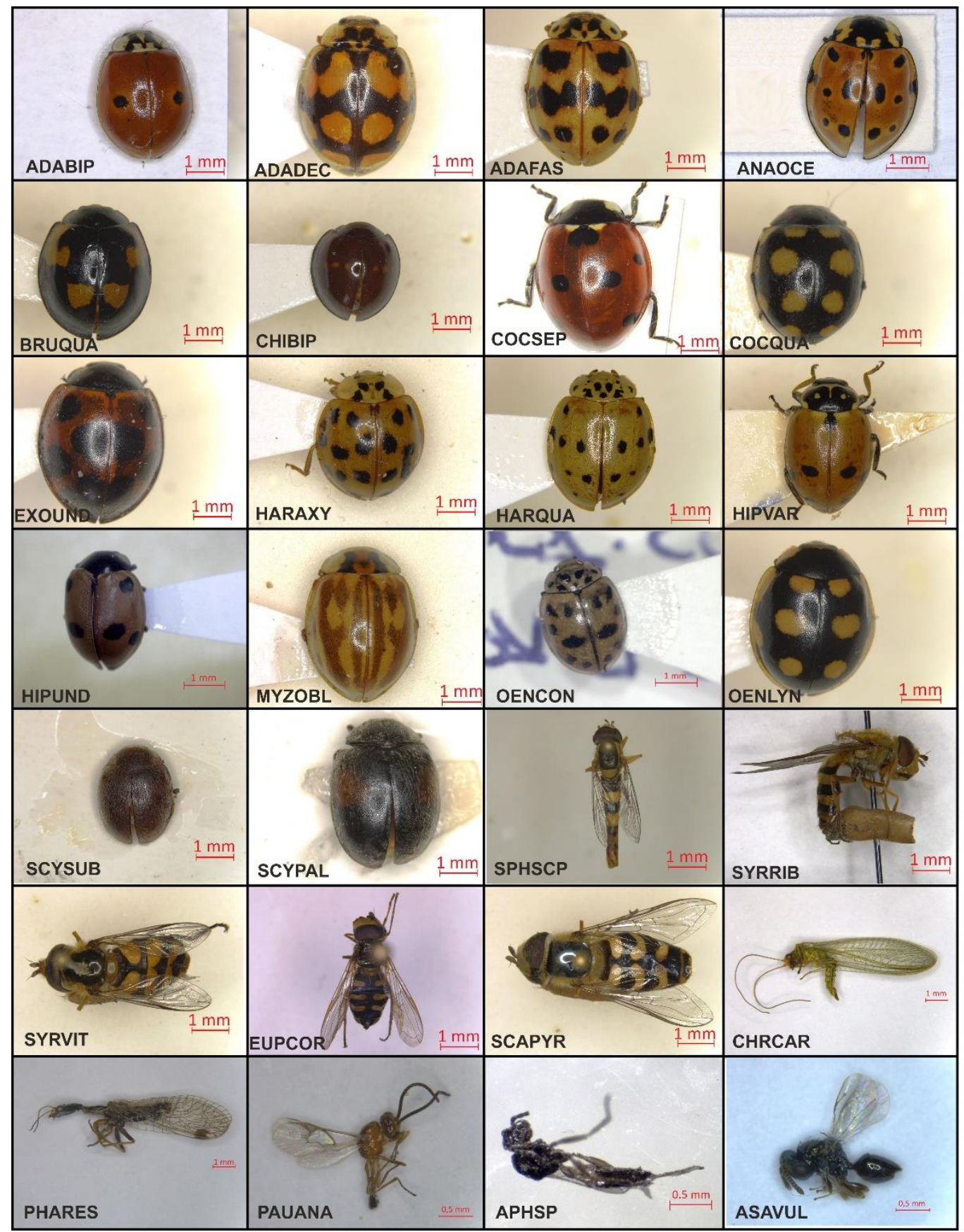

Figure 3. Natural enemies species of Cinara cedri 
The highest number of species was found in the Coccinellidae (18 species). This work is the first to demonstrate that $A$. decempunctata, A. fasciopunctata revelierei, C. bipustulatus, E. undulatus, $C$. quatardecimpustulata, $H$. quadripunctata, $H$. undecimnotata, $M$. oblongoguttata, $O$. lyncea, S. pallipediformis and S. subvillosus are also predators of $C$. cedri. Five species in the Syrphidae were also recorded as predators of $C$. cedri for the first time. The discovery of Syrphus vitripennis was the first record for study area; moreover, it was the syrphid with the highest number of individuals. From the Chrysopidae, Chrysopa carnea was found for the first time as a predator of C. cedri. 4 adults of this species were identified. One individual of $P$. ressli (Raphidiidae) was identified in each of 2 plots during sampling, the first record of this insect as a predator of $C$. cedri. The most common natural enemy found was Pauesia anatolica (Braconidae). Two individuals of another braconid Aphidius sp., were also found. Asaphes vulgaris (Pteromalidae) emerged from mummified of $C$. cedri during incubation in the laboratory. A. vulgaris and $P$. anatolica were detected the first time in the study area (Figure 3).

Natural enemies were not detected in 4 of the areas examined (Isparta-Çobanisa, Isparta-Keçiborlu-1, Burdur-AltınyaylaTamtır and Burdur-Bucak-Seydiköy). The highest number of natural enemies, 176 individuals, was found on the Isparta-SDU Campus, Isparta-Center with 155 and IspartaAtabey with 127. In terms of number of species, 13 were found in both Isparta-Center and on the Isparta-SDU Campus, while 11 species were found in Isparta-Gönen. Although the number of individuals was higher in Isparta-Atabey than in IspartaGönen, only four species were found in Isparta-Atabey. It was only found one species for each of 7 study areas (Figure 4). The numbers of species found within study areas varied between 1 and 25. $P$. anatolica was found in 25 sites, $H$. quadripunctata in 20 sites, and C. septempunctata in 16 sites. Nine natural enemies determined only in one area (Figure 5).
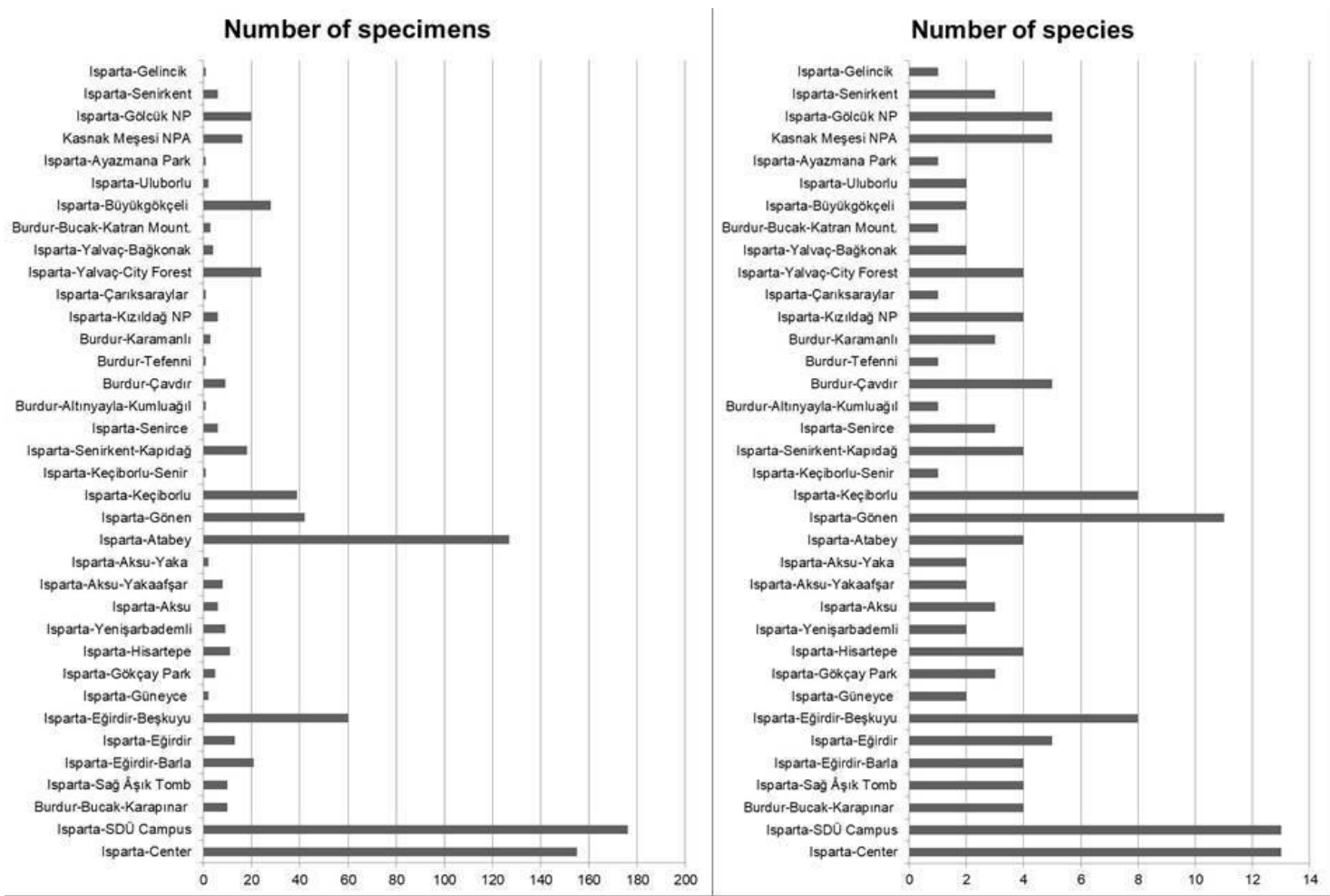

Figure 4. The number of species and specimens of Cinara cedri in study areas 


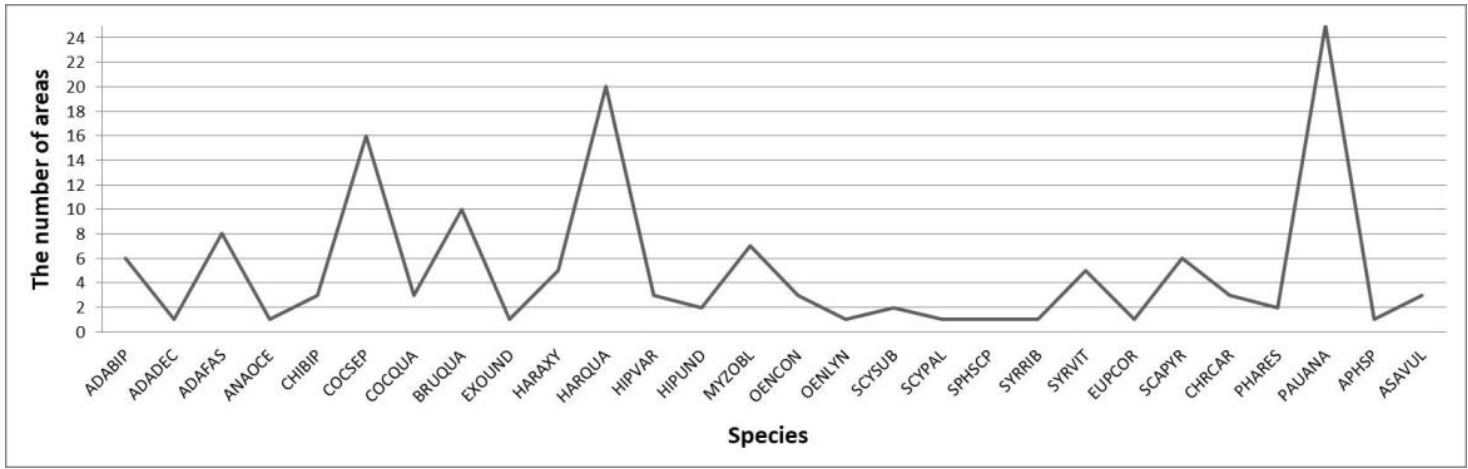

Figure 5. The number of areas where natural enemies of Cinara cedri were found

Locations, where the natural enemies were found during this work, along with dates of collection and numbers of individuals recovered are presented below.

\section{Predators}

Adalia bipunctata (Linnaeus, 1758)

(Coleoptera: Coccinellidae)

Material examined. Isparta-Center, 03.04.2016, (4); 09.04.2016, (2); IspartaSDÜ Campus, 04.04.2016, (1); 08.04.2016, (5); 19.05.2016, (1); 20.05.2016, (1); 28.05.2016, (1); Isparta-Gökçay Park, 02.06.2016, (1); Isparta-Hisartepe, 02.06.2016, (1); Isparta-Keçiborlu-2, 15.06.2016, (3); Isparta-Eğirdir-Beşkuyu, 10.08.2016, (1) (Total 21 specimens).

\section{Adalia decempunctata (Linnaeus, 1758)}

Material examined. Isparta-Center, 03.04.2016, 1 specimen.

\section{Adalia fasciatopunctata revelierei Mulsant \\ Material examined. Isparta-Center,} 02.11.2015, (1); 03.04.2016, (8); 09.04.2016, (1); Isparta-SDÜ Campus, 04.04.2016, (4); 08.04.2016, (5); 19.05.2016, (4); 20.05.2016, (1); 28.05.2016, (3); 09.09.2016, (1); IspartaHisartepe, 02.06.2016, (2); IspartaKeçiborlu-2, 15.06.2016, (2); Isparta-Gönen, 15.06.2016, (1); Isparta-Gölcük Naturel Park, 11.08.2016, (1); Isparta-Ayazmana Park, 21.08.2016, (1); Isparta-Senirkent, 12.10.2016, (2) (Total 37 specimens).

Anatis ocellata (Linnaeus, 1758)

Material examined. Isparta-Gölcük Naturel Park, 15.07.2016, 3 specimens.

\section{Chilocorus bipustulatus (Linnaeus, 1758)}

Material examined. Isparta-Center, 09.04.2016, (1); Isparta-Eğirdir, 29.04.2016, (3); 03.09.2016, (1); Isparta-SDÜ Campus, 18.08.2016, (1) (Total 6 specimens).

\section{8 \\ Coccinella septempunctata Linnaeus,}

Material examined. Isparta-Atabey, 15.06.2016, (72); Isparta-Keçiborlu-2, 15.06.2016, (7); Isparta-Gönen, 15.06.2016, (6); Isparta-Senirkent-Kapıdağ, 20.06.2016, (9); Isparta-Senirce, 21.06.2016, (3); BurdurKaramanl1, 22.06.2016, (1); Burdur-Çavdır, 22.06.2016, (2); Burdur-AltınyaylaKumluağıl, 22.06.2016, (1); Isparta-YalvaçBağkonak, 11.07.2016, (1); Isparta-Kızıldağ National Park, 11.07.2016, (1); IspartaYalvaç City Forest, 11.07.2016, (7); IspartaGelincik, 15.07.2016, (1); Isparta-Gölcük Naturel Park, 15.07.2016, (6); 11.08.2016, (3); Burdur-Bucak-Katran Mountain, 27.07.2016, (3); Isparta-Eğirdir-Beşkuyu, 10.08.2016, (34); Isparta-Eğirdir-Kasnak Meşesi Nature Reserve Area, 28.09.2016, (11) (Total 168 specimens).

Coccinula

(Linnaeus, 1758)

quatardecimpustulata

Material examined. Isparta-Kızıldağ National Park, 11.07.2016, (1); IspartaGölcük Naturel Park, 11.08.2016, (2); Isparta-Eğirdir-Kasnak Meşesi Nature Reserve Area, 18.09.2016, (1) (Total 4 specimens).

\section{Brumus quadripustulatus Linnaeus, 1758}


Material examined. Burdur-BucakKarapınar, 08.11.2015, (1); Isparta-Center, 03.04.2016, (5); 09.04.2016, (1); IspartaSDÜ Campus, 04.04.2016, (3); 08.04.2016, (5); 19.05.2016, (3); 20.05.2016, (1); 28.05.2016, (2); Isparta-Sağ Âş1k Tomb, 07.04.2016, (1); Isparta-Eğirdir-Barla, 21.04.2016, (3); Isparta-Eğirdir, 29.04.2016, (1); Isparta-Eğirdir-Beşkuyu, 31.05.2016, (1); Isparta-Atabey, 15.06.2016, (13); 07.04.2016, (1); 24.05.2016, (5); 07.05.2016, (4); Burdur-Çavdır, 22.06.2016, (2); BurdurKaramanl1, 22.06.2016, (1) (Total 53 specimens).

\section{Exochomus undulatus Weise, 1878}

Material examined. Burdur-BucakKarapınar, 08.11.2015, 1 specimen.

\section{Harmonia axyridis Pallas, 1773}

Material examined. Isparta-Center, 09.04.2016, (1); Isparta-SDÜ Campus, 19.05.2016, (1); Isparta-Gönen, 15.06.2016, (1); Isparta-Keçiborlu-2, 15.06.2016, (1); Isparta-Uluborlu, 05.08.2016, (1) (Total 5 specimens).

\section{Harmonia \\ (Pontoppidan, 1763) \\ Material examined. Isparta-Center,} 03.04.2016, (26); 28.04.2016, (1); 07.05.2016, (9); Isparta-Eğirdir, 29.04.2016, (1); 01.05.2016, (2); Isparta-Eğirdir-Barla, 24.05.2016, (1); 17.09.2016, (1); BurdurBucak-Karapinar, 07.04.2016, (1); 24.05.2016, (6); Isparta-Güneyce, 31.05.2016, (1); Isparta-Sağ Âş1k Tomb, 10.05.2016, (1); Isparta-SDÜ Campus, 04.04.2016, (4); 08.04.2016, (2); 12.04.2016, (1); 18.04.2016, (1); 19.05.2016, (12); 20.05.2016, (27); 28.05.2016, (19); IspartaEğirdir-Beşkuyu, 31.05.2016, (2); IspartaKeçiborlu-2, 15.06.2016, (9); Isparta-Yalvaç City Forest, 11.07.2016, (1); Isparta-Atabey, 15.06.2016, (27); Isparta-Aksu, 09.06.2016, (1); Isparta-Senirce, 16.06.2016, (1); 21.06.2016, (1); Isparta-Gönen, 15.06.2016, (11); Isparta-Hisartepe, 02.06.2016, (5); Burdur-Çavdır, 22.06.2016, (1); IspartaUluborlu, 05.08.2016, (1); Isparta-Gölcük Naturel Park, 11.08.2016, (5); IspartaKızıldağ National Park, 11.07.2016, (1);
Isparta-Senirkent, 12.10.2016, (3) (Total 185 specimens).

\section{Hippodamia (Hippodamia) variegata Goeze, 1777 \\ Material examined. Isparta-Gönen, 15.06.2016, (1); Burdur-Karamanl, 22.06.2016, (1); Isparta-Eğirdir-Beşkuyu, 10.08.2016, (3) (Total 5 specimens).} 1792)

Hippodamia undecimnotata (Schneider,

Material examined. Isparta-Gönen, 15.06.2016, (1); Isparta-Keçiborlu-2, 15.06.2016, (1) (Total 2 specimens).

Myzia oblongoguttata (Linnaeus, 1758)

Material examined. Isparta-Hisartepe, 02.06.2016, (3); Isparta-Senir, 16.06.2016, (1); Isparta-Senirkent-Kapıda ğ, 20.06.2016, (1); Isparta-SDÜ Campus, 21.06.2016, (1); Isparta-Çarıksaraylar, 11.07.2016, (1); Isparta-Ĕ̆irdir-Kasnak Meşesi Nature Reserve Area, 28.09.2016, (1); IspartaEğirdir-Beşkuyu, 10.08.2016, (1) (Total 9 specimens).

Oenopia conglobata (Linnaeus, 1758)

Material examined. Isparta-Eğirdir, 02.05.2016, (2); Isparta-Center, 07.05.2016, (2); Isparta-SDÜ Campus, 19.05.2016, (1) (Total 5 specimens).

\section{Oenopia lyncea (Olivier, 1808)}

Material examined. Isparta-EğirdirKasnak Meşesi Nature Reserve Area, 28.09.2016, 2 specimens.

\section{Scymnus subvillious (Goeze, 1777)}

Material examined. Isparta-Yalvaç City Forest, 11.07.2016, (9); IspartaBüyükgökçeli, 02.08.2016, (14) (Total 23 specimens).

\section{Scymnus pallipediformis Günther, 1958}

Material examined. Isparta-EğirdirBeşkuyu, 10.08.2016, 1 specimen.

Sphaerophoria scripta (Linnaeus, 1758) (Diptera: Syrphidae)

Material examined. Isparta-Atabey,

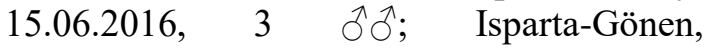
15.06.2016, 2 $\widehat{ึ}$ (Total 5 specimens). 
Syrphus ribesii (Linnaeus, 1758)

Material examined. Isparta-SDÜ Campus, 19.05.2016, 1 ㅇ specimen.

\section{Syrphus vitripennis Meigen, 1822}

Material examined. Isparta-Sağ Âşık Tomb,

10.05.2016, 1 đ, 1 क; Isparta-EğirdirBarla, 24.05.2016, 5 ठํํ, 3 웅 IspartaAksu-Yakaafşar, 09.06.2016, $3 \hat{\jmath} \hat{\partial}, 2$ 오; Isparta-Aksu, 09.06.2016, $2 \hat{\partial} \bar{\partial}, 2$ 우우

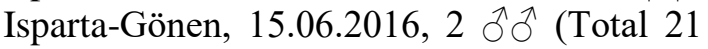
specimens).

\section{Eupeodes corollae (Fabricius, 1794)}

Material examined. Isparta-SDÜ Campus, 18.04.2016, 1 ㅇ, 1 त (Total 2 specimens).

\section{Scaeva pyrastri (Linnaeus, 1758)}

Material examined. Isparta-Center, 03.04.2016, 1 त; Isparta-SDÜ Campus,

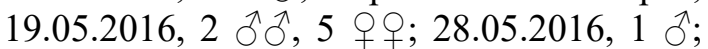
02.06.2016, 1 ㅇ ; 19.05.2016, 2 우우 IspartaAksu-Yaka, 09.06.2016, 1 गे; IspartaYenişarbademli, 09.06.2016, 1 $\hat{0}, 1$ क;

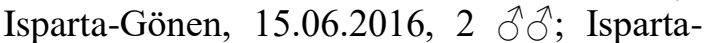
Keçiborlu-2, 15.06.2016, 1 त (Total 18 specimens).

Chrysoperla carnea (Stephens, 1836) (Raphidioptera: Chrysopidae)

Material examined. Isparta-Güneyce, 31.05.2016, (1); Isparta-Gönen, 15.06.2016, (1); Isparta-Center, 03.09.2016, (1); 0.10.2016, (1) (Total 4 specimens).

Phaeostigma (Aegeoraphidia) ressli (Aspöck \& Aspöck, 1964) (Raphidioptera: Raphidiidae)

Material examined. Isparta-SenirkentKapıdağ, 20.06.2016, (1); Burdur-Çavdır, 22.06.2016, (1) (Total 2 specimens).

\section{Parasitoids}

Pauesia (Pauesia) anatolica Michelena, Assael \& Mendel, 2005 (Hymenoptera: Braconidae)

Material examined. Isparta-Center, 03.04.2016, (21); 23.04.2016, (9); 29.04.2016, (1); $\quad 01.05 .2016, \quad$ (16); 02.05.2016, (16); 07.05.2016, (11); 07.10.2016, (4); Isparta-Sağ Âşık Tomb,
07.04.2016, (6); Isparta-Barla, 21.04.2016, (1); 24.05.2016, (1); 17.09.2016, (6); IspartaSDÜ Campus, 19.05.2016, (3); 02.06.2016, (17); 28.05.2016, (2); 02.06.2016, (16); 18.08.2016, (12); Isparta-Eğirdir, 02.05.2016, (1); 02.08.2016, (2); BurdurBucak-Karapınar, 24.05.2016, (1); IspartaEğirdir-Beşkuyu, 31.05.2016, (17); IspartaGönen, 15.06.2016, (13); Isparta-Gökçay Park, 02.06.2016, (2); IspartaYenişarbademli, 09.06.2016, (7); IspartaAksu, 09.06.2016, (1); Isparta-Keçiborlu-2, 15.06.2016, (15); Isparta-Aksu-Yaka, 09.06.2016, (1); Isparta-Hisartepe, 02.06.2016, (2); Isparta-Senirkent-Kapıdağ, 20.06.2016, (7); Burdur-Çavdır, 22.06.2016, (3); Burdur-Tefenni, 22.06.2016, (1); IspartaAksu-Yakaafşar, 09.06.2016, (3); IspartaSenirce, 21.06.2016, (1); Isparta-Yalvaç City Forest, 11.07.2016, (7); Isparta-Kızıldağ National Park, 11.07.2016, (3); IspartaYalvaç-Bağkonak, 11.07.2016, (3); IspartaBüyükgökçeli, 02.08.2016, (14); IspartaSenirkent, 12.10.2016, (1); Isparta-EğirdirKasnak Meşesi Nature Reserve Area, 05.10.2016, (1) (Total 248 specimens).

\section{Aphidius sp.}

Material examined. Isparta-Center, 03.04.2016, 2 specimens.

\section{Asaphes vulgaris Walker, 1834 (Hymenoptera: Pteromalidae) \\ Material examined. Isparta-Center, 03.04.2016, (6); 23.04.2016, (3); Isparta- Gönen, 15.06.2016, (3); Isparta-SDÜ Campus, 28.05.2016, (1) (Total 13 specimens).}

\section{Discussion}

In this work, 28 natural enemies of $C$. cedri were found in the Isparta Regional Forest Directorate, including 18 coccinellid (Coleoptera), 5 syrphid (Diptera), 2 braconid, 1 pteromalid (Hymenoptera), 1 raphidiid and 1 chrysopid (Neuroptera). The highest number of species was identified from the Coccinellidae. An invasive alien species, $H$. axyridis was newly recorded in study area, underlying the importance of this work in terms of detection and distribution of this species in Turkey. 
Adalia bipunctata was already reported as a predator of $C$. cedri in previous studies (Núñez-Pérez \& Tizado, 1996; Karaca et al., 2006; Glavendekić, 2012). In this study, 21 individuals of this species were recorded in 6 areas, although 6 individuals of this species had been reported in 3 areas in Isparta (Karaca et al., 2006). Oğuzoğlu, Avc1, Şenal \& Karaca, (2017) reported that A. ocellata, a predator of $C$. cedri, as a new record for Turkish fauna. Núňez-Perez \& Tizado (1996) reported that A. ocellata was a predator of $C$. pinea (Mordvilko, 1894), a species related to C. cedri. Coccinella septempunctata (Aslan \& Uygun, 2005; Glavendekić, 2012; Mouna, 2013; Aslan, 2014), B. quadripustulatus (Aslan \& Uygun, 2005; Mouna, 2013), $H$. variegata (Mouna, 2013; Aslan, 2014) and O. conglobata (Aslan \& Uygun, 2005; Aslan, 2014) are also known predators of $C$. cedri. Glavendekić (2012) stated that $H$. axyridis was one of the most common natural enemies of C. cedri in Belgrade, Serbia.

Five species of Syrphidae as predators of C. cedri were found for the first time. Syrphus vitripennis is the first record for Isparta province and had the highest number of individuals among the other Syrphidae species found. Sphaerophoria scripta (Linnaeus, 1758) was reported on Rosa damascena in Isparta-Center, Gönen, and Keçiborlu by Demirözer \& Karaca (2014), in Burdur, Isparta-Merkez, Yalvaç, SenirkentKapıdağ and on Isparta-Antalya road by Sarıbıyı \& Hasbenli (2006). In this study, the species was found in Atabey and Gönen. Sarıbıyık \& Hasbenli (2006) found S. ribesii in Isparta, Yalvaç-Senirkent road and Yalvaç. In this study, this species was found on the campus of Isparta-SDU. Aslan (2015) recorded that Sryphus vitripennis was a predator of Dysaphis devecta (Walker, 1849) (Hemiptera: Aphididae) in Burdur. Eupeodes corellea (Fabricius, 1794) was found on Rosa damascena in Isparta-Center, Eğirdir and Keçiborlu by Demirözer \& Karaca (2014), in Burdur, Isparta-Antalya road and Yalvaç by Sarıbıyık \& Hasbenli (2006), and in Burdur by Aslan (2015), whereas in the present work, the species was found on Isparta-SDU Campus. Scaeva pyrasti (Linnaeus, 1758) was reported in SenirkentKapıdağ, Gelendost, Yalvaç and Isparta-
Center by Sarıbıyık \& Hasbenli (2006). In contrast, shoots with $C$. cedri collected from Gelendost had no S. pyrasti present. Aslan (2014) stated that Episyrphus balteatus (De Geer) and Meliscaeva auricollis Meigen (Diptera: Sryphidae) were predators of $C$. cedri in Kahramanmaraş, Turkey, but these species were not found in the present work.

Canbulat (2003) reported that Chrysopa (=Chrysoperla) carnea was found on $C$. libani in Isparta-Senirkent, KeçiborluÖzbahçe, Eğirdir, Isparta-Center, Çarıksaraylar and Isparta-Güneyce. $C$. carnea was found in the same sites (except Keçiborlu-Özbahçe) in the present work. Glavendekić (2012) stated that Chrysopa sp. was a common natural enemy of $C$. cedri. As a predator of $C$. cedri, Hemerobius micans Olivier, 1792 (Neuroptera: Hemerobiidae) was stated by Tosun (1975) and Usta \& Keskin (1992) but was not found in this work. In our study, Phaeostigma ressli (Raphidiidae) was observed in two sites and recorded as a predator of $C$. cedri for the first time. Canbulat (2003) reported that this species was observed on C. libani and it was distributed in Burdur-Çavdır. Two samples were collected in June in the present work, whereas Canbulat (2003) found this species in April and July.

Pauesia anatolica was identified for the first time in the provinces of Isparta and Burdur, and it was the species with the highest number of individuals found in the study. This species was defined as Pauesia cedri in 1991 in Turkey (Mouna \& Fabre, 2005). Pauesia sp. which is a parasitoid of $C$. cedri was recorded for the first time by Aslan, Uygun \& Starý (2004) in 1999-2001 in the natural distribution area of $C$. libani. In 2002, the species obtained from the mummies of $C$. cedri was defined by Michelena, Assael \& Mendel (2005) as $P$. anatolica and noted as a parasitoid of $C$. cedri. Aytar (2006) found C. cedri on $C$. libani in Adana, Ankara, Karaman, Mersin, Niğde, Osmaniye provinces in 2002-2006. The present study found two individuals of Aphidius sp. There are no studies in the literature showing that it is a parasitoid of $C$. cedri. However, it was stated previously that Aphidius species is a parasitoid of conifer aphids (Cinara spp.) (Watanabe, 1941). 
Asaphes vulgaris, a parasitoid of C. cedri, was identified in the Isparta and Burdur for the first time in the present work. According to literature, A. vulgaris is hyperparasitoid of parasitoids (Pauesia sp.) of Cinara species (Müller, Völkl \& Godfray, 1997).

The research is the first detailed study about natural enemies of $C$. cedri in Turkey forests. There is one only study about natural enemies of $C$. cedri in forest in the southeast of Anatolia and in this study, it was found one species, Pauesia anatolica (Aytar, 2006).

\section{Conclusions}

Although C. cedri was frequently found in this study in the cedar forests of Isparta Regional Forest Directorate, there were no signs of significant damage except to a few areas. The presence of abundant natural enemies in this area could explain the low amount of damage that was present. Natural enemies were determined in $90 \%$ of sampling sites and the richness and numbers of natural enemy species in many sites were particularly notable. It is suggested here that protection of mixed forests will increase biological diversity and efficacy of natural enemies in forests. C. cedri seems not to need chemical control in mixed forests due to the large amount and variety of natural enemies (Coleoptera, Diptera, Hymenoptera and Neuroptera). If the population of C. cedri increases, these natural enemies could be used as a biological control agency. In sites damaged by $C$. cedri, natural enemies can be transferred into the area if predatory populations are inadequate and lack diversity. Further studies related to natural enemy species and the possibilities of their usage within the scope of biological control programs should be investigated. But, chemical control (especially biopesticides) may be applied in heavily infested sites in park and gardens, in order to eliminate visual damage due to intensive honeydew secretion and sooty-mold growth.

\section{Acknowledgements}

This study was part of the MSc dissertation of the first author accepted by SDU Graduate School of Natural and Applied Sciences on 10 January 2017. We would like to thank Prof. Dr. İsmail
KARACA (Isparta University of Applied Sciences, Faculty of Agriculture, Plant Protection Department) for contributions. We would like to thank Prof. Dr. Nedim UYGUN (Çukurova University, Faculty of Agriculture, Plant Protection Department Retired Faculty Member-Adana) and Assis. Prof. Derya ŞENAL (Bilecik Şeyh Edebali University, Faculty of Agriculture and Natural Sciences, Plant Protection Department) for coccinellid preparation and identification; Prof. Dr. Rüstem HAYAT (Süleyman Demirel University, Faculty of Agriculture, Plant Protection Department) for the identification of Syrphidae samples, Prof. Dr. Savaş CANBULAT (Kastamonu University, Faculty of Engineering and Architecture, Environmental Engineering Department) for the identification of Chrysopidae and Raphidiidae, Prof. Dr. Miktat DOĞANLAR (Mustafa Kemal University, Faculty of Agriculture, Plant Protection Department Retired Faculty Member) for the identification of Braconidae and Pteromalidae and Suleyman Demirel University Academic Training Program Coordination Unit for its support for our project numbered ÖYP-06475-YL15.

\section{References}

Akyıldırım, H., Şenol, Ö., Görür, G. \& Demirtaş, E. (2013). Evaluation of the Zoogeographical Contents of Turkey aphid (Hemiptera, Aphidoidea) fauna and Invasive components. Biyoloji Bilimleri Araştırma Dergisi, 6(1), 44-48.

Aslan, M. M., Uygun, N. \& Starý, P. (2004). A survey of aphid parasitoids in Kahramanmaras, Turkey (Hymenopteraa: Braconidae, Aphidiinae; and Hymenoptera: Aphelinidae). Phytoparasitica, 32(3), 255263.

Aslan, M. M. \& Uygun, N. (2005). The aphidophagus coccinellid (Coleoptera: Coccinellidae) species in Kahramanmaraş, Turkey. Turkish Journal of Zoology, 29, 1-8.

Aslan, M. M. (2014). Kahramanmaraş ili ağaçlarında Aphidoidea (Hemiptera) türleri ve doğal düşmanları. Türkiye II. Orman Entomolojisi ve Patolojisi Sempozyumu, (pp. 456-466), 7-9 Nisan, Antalya, (in Turkish).

Aslan, B. (2015). Survey of the predatory and parasitoid species-complex of aphids (Hemiptera: Aphididae) at fruit growing areas 
in Burdur, Turkey. Egyptian Journal of Biological Pest Control, 25(1), 261-265.

Aytar, F. (2006). Geographical distribution of Cinara cedri, Cinara (Cedrobium) laportei (Homo.; Aphididae) and newly discovered a parasitoid of Cinara cedri, Pauesia (Pauesia) anatolica (Hym.; Braconidae) in Turkey. VIII The European Congress of Entomology, 1722 September 2006, İzmir, Turkey, p. 27.

Blackman, R.L. \& Eastop, V.F. (2012). Aphid's on the World's Trees. An Identification and Information Guide CAB International, Wallingford, $1004 \mathrm{p}$.

Canbulat, S. (2003). Raphidiopter's and Neuropter's (Insecta, Neuropterida) South West Anatolia. Gazi University, Institute of Science and Technology, PhD Thesis, 331 p., Ankara. (in Turkish with English abstract).

Çanakçığlu, H. (1975). The Aphidoidea of Turkey. İstanbul University Forest Faculty Publications, İstanbul University Publication No: 1751, Forest Faculty Publication No: 189, 309p.

Demirözer, Ö. \& Karaca, İ. (2014). Predator and parasitoid species associated with oil-bearing rose (Rosa damascena Miller) production areas in Isparta province with distributional remarks. Turkish Bulletin of Entomology, 4(3), 171-184. (in Turkish with English abstract)

Düzgüneş, Z., Toros, S., Kılınçer, N. \& Kovanc1, B. (1980). Ankara İlinde Saptanan Afit Predatörleri ve Bunların Biyolojik Mücadelede Kullanılma Olanaklar1. TÜBİTAK-TOAG Project Report, Project No: 295, 198p (in Turkish).

Glavendekić, M. (2012). Integrated pest management of insects in urban green spaces. In International Symposium: Current Trends in Plant Protection-Proceedings, 25-28 September, Belgrade, Serbia, pp. 451-454.

Karaca, İ., Karsavuran, Y., Avcı, M., Demirözer, O., Aslan, B., Sökeli, E. \& Bulut, H. S. (2006). Faunistic Studies on Coleoptera Species of Isparta Province (Turkey). Süleyman Demirel University Journal of Natural and Applied Sciences, 10(2), 180184. (in Turkish with English abstract).

Michelena, J.M., Assael, F. \& Mendel, Z. (2005). Description of Pauesia (Pauesia) anatolica (Hymenoptera: Braconidae, Aphidiinae) sp. nov., a parasitoid of the cedar aphid Cinara cedri. Phytoparasitica, 33(5), 499-505.

Mimeur, J.M. (1935). Aphididae Du Maroc (Septiéme Note). Bulletin de la Société des Sciences Naturelles du Maroc Xv, (3), 251258.
Mouna, M. (2013). Les insectes du cèdre de l'Atlas (Cedrus altantica Manetti) dans le Nord de 1'Afrique. Travaux de I'Institut Scientifique Rabat, Série Zoologie, 48, 1-89.

Mouna, M. \& Fabre, J.P. (2005). Pest Insects of Cedars: Cedrus atlantica Manetti, C. libani A. Richard and $C$. brevifolia Henry in the Mediterranean Area. F., Lieutier, D., Ghaioule (Eds), Entomological research in Mediterranean forest ecosystems (89-104), Institut National de la Recherche Agronomique, Paris, 275 pp.

Müller, C.B., Völkl, W. \& Godfray, C. J. (1997). Are behavioural changes in parasitised aphids a protection against hyperparasitism? European Journal of Entomology, 94, 221234.

Núňez-Perez, E. \& Tizado, E. J. (1996). Conifer aphids (Homoptera: Aphididae) and some natural enemies in the León province (Spain). Boletín de la Asociación española de Entomología, 20(1-2), 85-93.

Orman Genel Müdürlüğü (OGM). (2015). Türkiye Orman Varlı̆̆ 2015 . Ankara: T.C. Orman ve Su İşleri Bakanlığı Orman Genel Müdürlüğü Yayını, 36s. (in Turkish).

Oğuzoğlu, Ş., Avc1, M., Şenal, D. \& Karaca, İ. (2017). First record of Anatis ocellata (Linnaeus, 1758) (Coleoptera: Coccinellidae) in Turkey. Turkish Bulletin of Entomology, 17(2), 197-202. DOI: 10.16969/entoteb.331402

Sarıbıyı,, S. \& Hasbenli, A. (2006). Fauna of Syrphinae and Milesiinae (Diptera: Syrphidae) in the Region of South-West Anatolia. Journal of the Entomological Research Society, 8(1), 43-52.

Steiner, H. (1962). Metoden zur Untersuchungen des Population dynamik in obstanlangen. Entomophaga, 7, 207-214.

Toper Kaygın, A., Görür, G. \& Çota, F. (2008). Contribution to the Aphid (Homoptera: Aphididae) Species Damaging on Woody Plants in Bartin, Turkey. International Journal of Natural and Engineering Sciences, 2(1), 83-86.

Tosun, İ. (1975). Akdeniz Bölgesi İğne Yapraklı Ormanlarında Zarar Yapan Böcekler ve Önemli Türlerin Parazit ve Yırtıcilarl Üzerine Araştırmalar. Orman Bakanlığı Orman Genel Müdürlüğü Yayınları Sıra No: 612 Seri No: 24, Vural Matbaas1, 201 s. (in Turkish).

Tuatay, N. (1999). Türkiye yaprakbitleri (Homoptera: Aphididae): V. Chaitophinae, Lachninae ve Thelaxinae. Bitki Koruma Bülteni, 39(1-2), 1-21. (in Turkish). 
Tuatay, N. \& Remaudiere, G. (1964). Premiere Contribution au Catalogue des Aphididae (Hom.) de la Turquie. Revue de Pathologie Vegetale Et Entomologie Agriculture de Paris, 43(4), 243-278.

Usta, H. Z. \& Keskin, S. (1992). Sedir Ormanlarının Zararlılarl ve Mücadelesi. Sedir El Kitabı Dizisi: 6. (Editör Ünal ELER) Ankara: Ormancılık Araştırma Enstitüsü Yayınları. Muhtelif Yayınlar Serisi: 66, 265286. (in Turkish).

Uygun, N., Toros, S., Ulusoy, R., Satar, S. \& Özdemir, I. (2000). Doğu Akdeniz Bölgesi Aphidoidea (Homoptera) Türleri ile Parazitoit ve Predatörlerinin Saptanması. Tübitak Proje Sonuç Raporu, Proje No: TOGTAG-1720, 215 s. (in Turkish).

Ülgentürk, S., Özdemir, I., Kozar, F., Kaydan, M. B., Dostbil, Ö., Sarıbaşak, H. \& Civelek, H. S. (2013). Honeydew producing insect species in forest areas in Western Turkey. Turkish Bulletin of Entomology, 3(4), 125133.

Ünal, S. \& Özcan, E. (2005). The Aphididae (Homoptera) species of Kastamonu Region. Süleyman Demirel Üniversitesi Orman Fakültesi Dergisi Seri A, 1, 76-83. (in Turkish with English abstract).

Watanabe, C. (1941). Descriptions of Three New Species of Aphidius Parasitic on Some Aphids of Coniferous Trees (Taxonomic Notes on Aphidiidae of Japan, 3). Insect Matsumurana, 15(3), 106-111. 